\title{
Influencia del tiempo de tratamiento de superficie con ácido fluorhídrico de la porcelana VITA VM 13 en la resistencia de unión a cemento de resina frente a fuerzas de tracción. Estudio in vitro
}

\author{
Influence of surface treatment time with hydrofluoric acid of VITA \\ VM13 porcelain on tensile bond strength to a luting resin cement. \\ In vitro study
}

\author{
Guzmán Thoms JP${ }^{1}$, González Bustamante $\mathrm{H}^{2}$, Salgado Montoya $\mathrm{M}^{2}$
}

\begin{abstract}
RESUMEN
Introducción: Los procedimientos restauradores indirectos son comunes en la clínica diaria actual. Se debe tener cuidado especial en la cementación, pues la gran mayoría de los fracasos ocurren en la interfase diente/restauración. Por esto es indispensable un adecuado tratamiento de superficie de la restauración para lograr una adhesión óptima a la superficie dentaria. Materiales y Métodos: Treinta cilindros de porcelana VITA VM 13 con dimensiones de $5 \mathrm{~mm}$ de altura y $7 \mathrm{~mm}$ de diámetro fueron separadas aleatoriamente en 3 grupos $(\mathrm{n}=10)$ de acuerdo con los tratamientos recibidos. Grupo I (control) - sin acondicionamiento superficial; Grupo II - grabado con ácido fluorhídrico al 9.6\% por 1 minuto; Grupo III - grabado con ácido fluorhídrico al $9.6 \%$ por 2 minutos. Estas fueron probadas en cuanto a su resistencia de unión a la tracción en una máquina universal para pruebas (INSTRON modelo 4411, 3M). Los valores, expresados en MPa, fueron analizados estadísticamente a un nivel de significación del 5\%, usando el Análisis de la Varianza (ANOVA) de un factor. Resultados: En relación con la prueba de resistencia de unión a la tracción, se encontraron los siguientes valores medios para cada grupo: Grupo I (control) - x=3.35 MPa; Grupo II - x=4.64 MPa; Grupo III - x=3.80 MPa. Conclusiones: Según los resultados obtenidos, se puede concluir que el tratamiento de superficie con ácido fluorhídrico durante 1 minuto, promueve microrretenciones que aumentan significativamente los valores de resistencia a la tracción, que puede traducirse en un beneficio clínico mayor.
\end{abstract}

Rev. Clin. Periodoncia Implantol. Rehabil. Oral Vol. 5(3); 117-122, 2012.

Palabras clave: Porcelana feldespática, resistencia de unión, acondicionamiento de superficie, grabado con ácido fluorhídrico.

\section{ABSTRACT}

Aim: Indirect restorative procedures are common in the current daily practice. Special care must be taken in the cement, as the vast majority of failures occurs at the interface tooth /restoration. Therefore a proper surface treatment of the restoration is essential to achieve optimum adhesion to the tooth surface. Materials and Methods: Thirty porcelain cylinders VITA VM 13 with dimensions of $5 \mathrm{~mm}$ in height and $7 \mathrm{~mm}$ in diameter were randomly divided into 3 groups $(n=10)$ according to the treatments received. Group I (control) - no surface conditioning; Group II- recorded with $9.6 \%$ hydrofluoric acid for 1 minute; Group III - recorded with $9.6 \%$ hydrofluoric acid for 2 minutes. Their tensile bond strength was tested in a universal testing machine (Instron Model 4411, 3M). The values, expressed in MPa, were statistically analyzed at a level of significance of $5 \%$, using Analysis of Variance (ANOVA) of a factor. Results: In relation to the bond strength test, tensile strength, revealed the following values for each group: Group I (control) - x=3.35 MPa, Group II - x=4.64 MPa, Group III - x=3.80 MPa. Conclusions: According to the results, it can be concluded that the surface treatment with hydrofluoric acid for 1 minute promotes microretentions, which significantly increase the values of bond strength, tensile strength. This can result in a higher clinical benefit.

Rev. Clin. Periodoncia Implantol. Rehabil. Oral Vol. 5(3); 117-122, 2012.

Key words: Feldspathic porcelain, bond strength, surface conditioning, hydrofluoric acid etching.

\section{INTRODUCCIÓN}

En la investigación odontológica, la síntesis de nuevos sistemas adhesivos multifuncionales con capacidad de unión a todo tipo de substratos ha sido una constante desde hace años, habiendo evolucionado los conceptos tradicionales, exigiendo al odontólogo nuevos conocimientos, y la adopción de la adhesión como elemento permanente y rutinario de su práctica diaria(1).

En odontología restauradora, los recursos adhesivos han experimentado cambios importantes, desde el acondicionamiento de la superficie dentaria iniciados por Buonocuore en 1955, con la técnica de grabar la superficie del esmalte con ácido fosfórico, hasta el grabado ácido de la porcelana, aporte de John Calamia y Harold Horn en 1983. De esta manera se abrió la posibilidad de adherir restauraciones cerámicas sobre las superficies dentarias ${ }^{(2)}$.

Un número creciente de los materiales y sistemas libres de metal están disponibles actualmente para uso clínico. Múltiples estudios clínicos a largo plazo documentan éxito de las restauraciones adheridas con cementos de resina, tales como carillas de porcelana ${ }^{(3-7)}$, inlays y onlays cerámicos ${ }^{(8-17)}$, prótesis fijas plurales ${ }^{(18-21)}$, y de coronas cerámicas $^{(8,22-24)}$.

Las restauraciones cerámicas se utilizan ampliamente, porque son durables, estéticamente atractivas, y proporcionan una excelente biocompatibilidad $^{(25)}$. Esta preferencia está directamente relacionada

1. Cirujano Dentista. Especialista en Rehabilitación Oral. Universidad San Sebastián. Chile.

2. Profesores Asociados. Facultad de Odontología, Universidad San Sebastián, Sede Santiago. Chile.

Correspondencia autor: Juan Pablo Guzmán Thoms. jp.guzman.t@gmail.com. Trabajo recibido el 03/04/2012. Aprobado para su publicación el 11/07/2012. 
con el éxito del enlace cemento de resina-cerámica que contribuye a la longevidad de la restauración. Para el tratamiento de superficie cerámica, el ácido fluorhídrico reacciona con la matriz de vidrio que contiene sílice y forma hexafluorosilicatos. Esta matriz de vidrio es selectivamente retirada y la estructura cristalina queda expuesta para la retención micromecánica de la cerámica ${ }^{(26,27)}$. Esta superficie grabada también ayuda a proporcionar más energía superficial antes de combinar con la solución de silano ${ }^{(28)}$. Por otro lado, el ácido fluorhídrico puede reducir la resistencia a la flexión de la cerámica ${ }^{(29)}$. Aunque algunos estudios han informado sobre el efecto de diferentes tiempos de grabado con ácido fluorhídrico en la fuerza de unión ${ }^{(27,28)}$, rugosidad ${ }^{(30)}$ y resistencia a la flexión ${ }^{(25,30)}$

La principal causa de fracaso en la cementación de prótesis fijas cerámicas se halla en la interfase cemento-cerámica ${ }^{(31)}$, es por ello que las investigaciones se orientan a conseguir una máxima unión a la cerámica. Esta unión puede ser a través de una adhesión mecánica y/o una adhesión química. De lo anterior se desprende la necesidad de preparar la superficie cerámica al momento de cementar una restauración libre de metal. Otra indicación clínica es cuando debemos realizar reparaciones de cerámicas fracturadas ${ }^{(31)}$.

El objetivo de modificar de la superficie de la porcelana antes de la cementación, es aumentar el área superficial disponible para la unión y para crear entalladuras que aumentan la resistencia de la unión al cemento de resina.

Una adhesión fuerte y duradera al cemento de resina proporciona una alta retención ${ }^{(32)}$, mejora la adaptación marginal, previene la microfiltración ${ }^{(33)}$, y aumenta la resistencia a la fractura del diente y la restauración ${ }^{(35)}$. Sin embargo, la composición y propiedades físicas de alta resistencia de los materiales cerámicos, tales como óxido de aluminio $(\mathrm{Al} 2 \mathrm{O} 3)^{(35-39)}$ y óxido de zirconio $(\mathrm{ZrO} 2)^{(40)}$, difiere sustancialmente de la cerámica a base de sílice ${ }^{(36,41,42)}$ y requieren de técnicas alternativas para lograr una unión fuerte a largo plazo. Los ensayos clínicos controlados son ideales para poner a prueba específica modalidades de tratamiento y su durabilidad a largo plazo. Sin embargo, las investigaciones in vitro son indispensables para identificar los materiales de alta calidad antes de su evaluación clínica, especialmente para los estudios comparativos de la unión agentes y cementos.

La adhesión a las cerámicas feldespáticas se obtiene normalmente por grabado la cerámica con ácido fluorhídrico para crear una superficie rugosa, favoreciendo la adherencia mecánica ${ }^{(43)}$. La unión también puede mejorarse mediante la aplicación de agentes de acoplamiento como el silano que son capaces de formar enlaces químicos tanto en la superficie inorgánica como la orgánica ${ }^{(44,45)}$. Una limitación potencial en el uso clínico de algún núcleo cerámica, como en el In Ceram por ejemplo, ha sido la incapacidad para grabar su superficie con ácido. Por esta razón, se han sugerido otros métodos y materiales para mejorar adhesión del cemento de resina a este material, tales como el uso de un recubrimiento de sílice-triboquímico (Rocatec, ESPE), con un chorro de arena o el uso de un cemento de resina modificado con un monómero de fosfato (Panavia 21, Kuraray) ${ }^{(46,47)}$.

El ácido fluorhídrico tiene afinidad para reaccionar con el sílice formando hexafluorsilicatos y, en cambio, no afecta en absoluto al óxido de aluminio. El resultado es una superficie que presenta el aspecto de un panal de abejas y ofrece retención micromecánica. Después se aplicará el silano y el sistema adhesivo. A pesar que se puede optar por el gel fluorfosfato acidulado, los valores de adhesión son inferiores a los obtenidos con ácido fluorhídrico (Della Bona y col. 2003)(48).

Las restauraciones de cerámica sin metal, son una alternativa importante, y muy difundida, dentro de los tratamientos odontológicos. Hay que resaltar que estas restauraciones requieren de una técnica, donde intervienen varios elementos que pueden determinar el éxito de dicha restauración. Dentro de estos elementos se pueden destacar, la capacidad de adhesión de la restauración cerámica a la superficie dentaria, sea esmalte o dentina, las características y preparación de la superficie cerámica utilizada, el tipo de cemento adhesivo, como los cementos de resina, y por último pero no menos importante el modo de polimerización de dichos cementos.

Con todo lo anterior, podemos afirmar que existen una serie de variables que intervienen en el procedimiento de cementación de los sistemas libres de metal. Uno de estos factores es el tiempo de grabado de la superficie cerámica con ácido fluorhídrico. La mayoría de los autores consultados en ese estudio concuerdan que el tiempo de grabado fluctúa entre 1 y 3 minutos, sugieriendo que 2 minutos es el tiempo óptimo de grabado, con el cual no se alteraría la composición de la mayoría de las cerámicas probadas. Tomando en cuenta lo anterior, se plantea la hipótesis de que la aplicación de ácido fluorhídrico por 2 minutos resulta en una mayor resistencia frente a fuerzas de tracción de las cerámicas VITA VM13, que su aplicación por 1 minuto al cementarlas con un cemento de resina. Los objetivos del presente estudio son analizar la influencia del tiempo de tratamiento de superficie con ácido fluorhídrico, en la resistencia a la tracción de las prótesis fijas libre de metal, estudiar la unión entre una cerámica, en la que se han utilizado distintos tiempos de tratamientos de superficie, a los cementos de resina, mediante un ensayo de tracción y realizar un estudio estadístico de los datos aportados en el ensayo de tracción con el fin de poder extraer conclusiones que pudieran indicarnos ciertas recomendaciones clínicas a la hora de cementar este tipo de restauraciones cerámicas.

\section{MATERIALES Y MÉTODOS}

Treinta cilindros de porcelana VITAVM 13 fueron confeccionados con las dimensiones de $5 \mathrm{~mm}$ de altura y $7 \mathrm{~mm}$ de diámetro. Los cilindros fueron incluidos en resina acrílica autopolimerizable de $30 \mathrm{~mm}$ de altura por $15 \mathrm{~mm}$ de ancho, a fin de dejar una cara expuesta de la cerámica para recibir el tratamiento superficial (Figura 1).

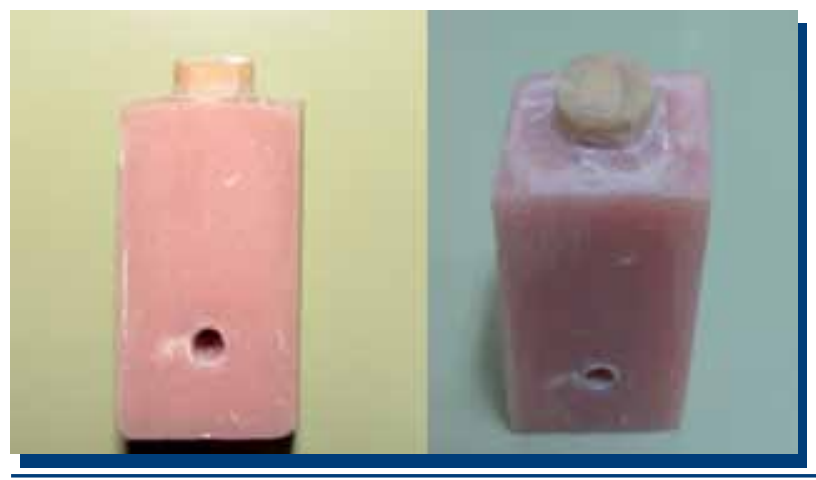

Figura 1. Muestra de cerámica incluida en cilindro de resina acrílica de autopolimerización.

Las muestras fueron separadas aleatoriamente en 3 grupos $(n=10)$ de acuerdo con los tratamientos recibidos. Los grupos probados fueron: Grupo I (control) - sin acondicionamiento superficial; Grupo II grabado con ácido fluorhídrico al $9.6 \%$ por 1 minuto; Grupo III - grabado con ácido fluorhídrico al $9.6 \%$ por 2 minutos.

Luego las muestras grabadas con ácido fluorhídrico (PorcelainEtch, Ultradent) fueron lavadas por 30 segundos con agua destilada y secadas con aire comprimido por 30 segundos (Figura 2)

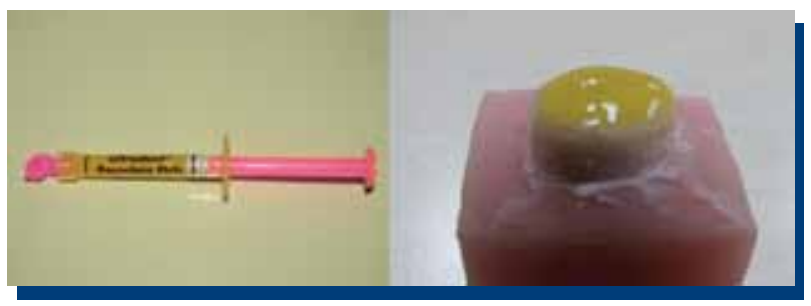

Figura 2. Ácido fluorhídrico, muestra acondicionada

Después de los respectivos acondicionamientos, a cada muestra de los grupos se le aplicó silano (Silano, Angelus) utilizándose para esto un pincel desechable y luego de 1 minuto, fueron secadas con chorro de aire por 5 segundos.

A continuación se colocó una huincha de celuloide para delimitar el área, con un diámetro interno de $7 \mathrm{~mm}$, a fin de estandarizar el área de unión y evitar el escurrimiento del cemento generando una mayor área de unión.

En un block para mezcla, se dispensaron iguales cantidades de pasta base y catalizadora del cemento con base de resina dual autoadhesivo (RelyX U100, 3M ESPE), se usó una espátula para mezclar la base y el catalizador para obtener una pasta homogénea. Después de mezclado, con ayuda de una espátula, el cemento fue colocado en la matriz de celuloide, con el propósito de confeccionar una base sobre la superficie tratada. Finalmente se fotopolimeriza con una lámpara 
halógena (Spectrum, Dentsply) a $400 \mathrm{~mW} / \mathrm{cm}^{2}$ por 20 segundos en cada cara.

Las muestras obtenidas fueron incluidas en un cilindro de resina acrílica de autopolimerización de $30 \mathrm{~mm}$ de altura y $15 \mathrm{~mm}$ de ancho para someterlas a la prueba de tracción (Figura 3).

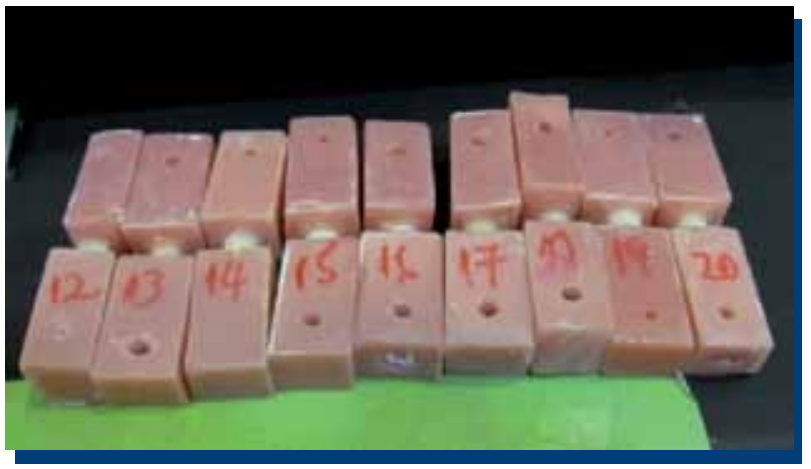

Figura 3. Muestras listas para ser sometidas a prueba de tracción.

Las muestras obtenidas, fueron almacenadas por 1 hora a temperatura ambiente y 23 horas en agua destilada a $37^{\circ} \mathrm{C}$. Después, éstas fueron probadas en cuanto a su resistencia de unión a la tracción en una máquina universal para pruebas (INSTRON modelo 4411, 3M) con velocidad de carga de $2 \mathrm{~mm} / \mathrm{min}$ (Figura 4).

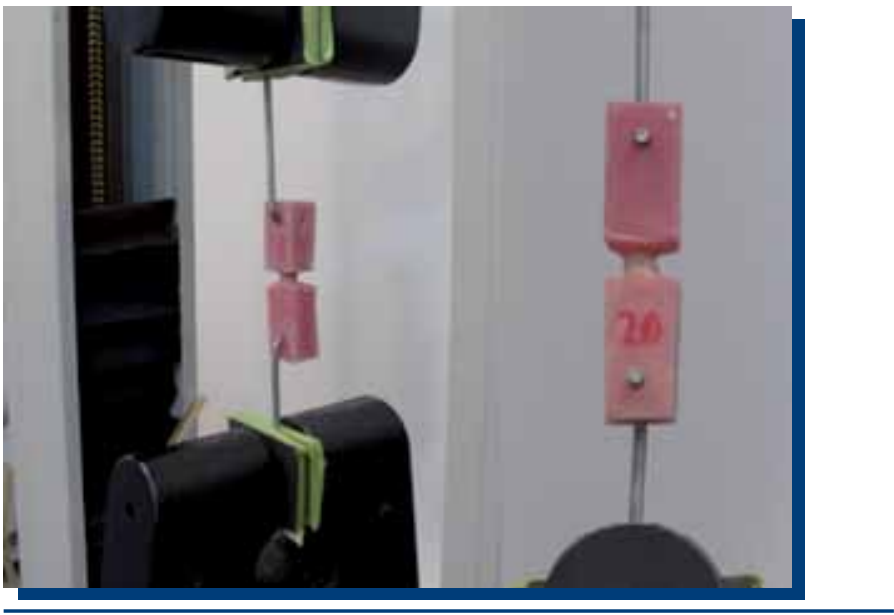

Figura 4. Muestras colocadas en las mordazas listas para ser traccionadas.

Los valores arrojados por la unidad de lectura de la máquina Instron fueron expresados en $\mathrm{KgF}$ para cada muestra probada. Posteriormente, los valores fueron transformados a MPa, usando la fórmula para calcular el área del círculo:

\section{Área del círculo $={ }_{\pi}{ }^{*} \mathbf{r}^{2}$}

Los valores obtenidos fueron analizados estadísticamente a un nivel de significación del $5 \%$, aplicando las siguientes pruebas estadísticas:

\section{Estadística descriptiva.}

Análisis de la Varianza (ANOVA) de un factor.

\section{RESULTADOS}

En relación con la prueba de resistencia de unión a la tracción, se encontraron los siguientes valores medios: Grupo I (control) - x=3.35 MPa; Grupo II - x=4.64 MPa; Grupo III - x=3.80 MPa, como se muestra en la Tabla 1.
Tabla 1. Resultados medios del análisis.

\begin{tabular}{|c|c|}
\hline Grupos & Media (MPa) \\
\hline I (control) & 3.35 \\
\hline II (ác. Fluorhídrico por 1 minuto) & 4.64 \\
\hline III (ác. Fluorhídrico por 2 minutos) & 3.80 \\
\hline
\end{tabular}

\section{Análisis Estadístico Descriptivo}

En la Tabla 2, se muestra las medias, medianas y modas de las fuerzas de unión, medidas en MPa, obtenidas para cada grupo, las desviaciones estándar, así como los valores máximos y mínimos.

Tabla 2. Estadística descriptiva.

\begin{tabular}{|c|c|c|c|c|c|c|c|c|c|c|c|}
\hline Grupo & $\begin{array}{c}\mathbf{N}^{\text {ro }} \\
\text { casos }\end{array}$ & Media & Mediana & Moda & $\begin{array}{c}\text { Modo } \\
\text { frecuencia }\end{array}$ & Suma & Mínimo & Máximo & Varianza & $\begin{array}{c}\text { Desv. } \\
\text { Estandar }\end{array}$ & $\begin{array}{c}\text { Eror } \\
\text { Estandar }\end{array}$ \\
\hline Control & 10 & 2.897 & 3.105 & Múltiple & 1 & 28.970 & 0.590 & 4.550 & 1.351 & 1.162 & 0.368 \\
\hline Ácido $1 \mathrm{~m}$ & 10 & 4.647 & 4.430 & Múltiple & 1 & 46.470 & 3.660 & 3.660 & 1.225 & 1.107 & 0.350 \\
\hline Ácido $2 \mathrm{~m}$ & 10 & 3.754 & 3.815 & Múltiple & 1 & 37.540 & 0.000 & 6.210 & 2.496 & 1.580 & 0.500 \\
\hline
\end{tabular}

La media más alta corresponde al Grupo II (ácido fluorhídrico 1 minuto) con un valor de resistencia a la tracción de $4.647 \mathrm{MPa}$, la media más baja está ubicada en el Grupo I (control), con un valor de resistencia a la tracción de $2.897 \mathrm{MPa}$.

Con respecto a los valores mínimos y máximos, en el Grupo III (ácido fluorhídrico 2 minutos), con un valor de $0 \mathrm{MPa}$, se observa el menor valor. El valor máximo se logró en el Grupo II, con un valor de resistencia a la tracción de $7.510 \mathrm{MPa}$.

En los Gráficos 1, 2 y 3 se representan la distribución de los resultados en diagramas de cajas (Boxplot), donde se puede observar la tendencia central, dispersión y asimetría, de los valores de resistencia a tracción obtenidos en cada grupo.

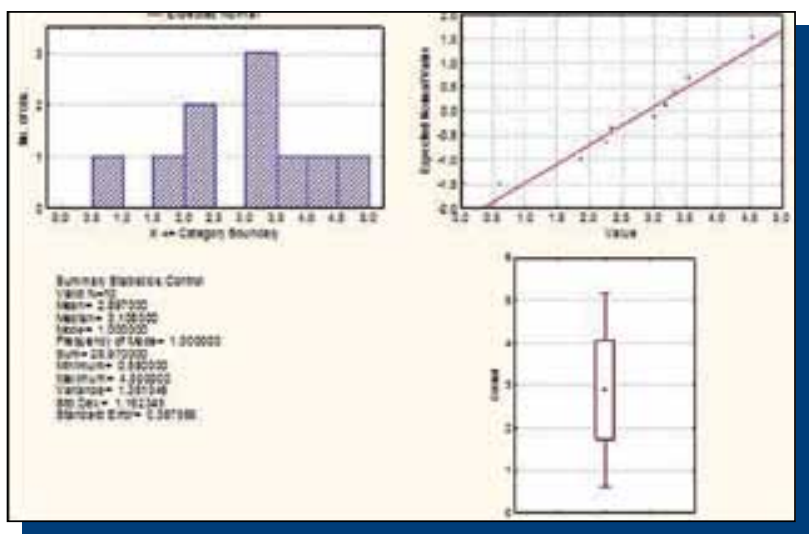

Gráfico 1. Histograma, distribución y Diagrama de Cajas (Boxplot) Grupo I.

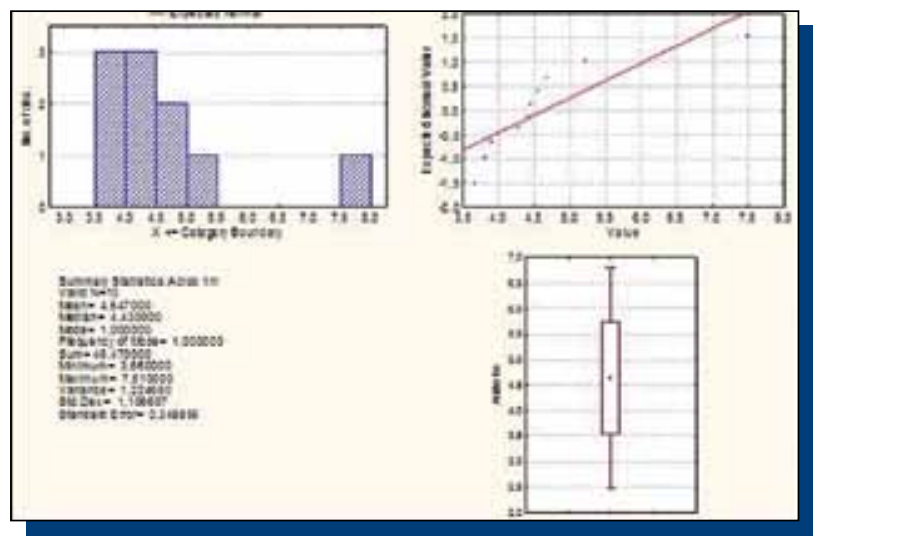

Gráfico 2. Histograma, distribución y Diagrama de Cajas (Boxplot) Grupo II. 


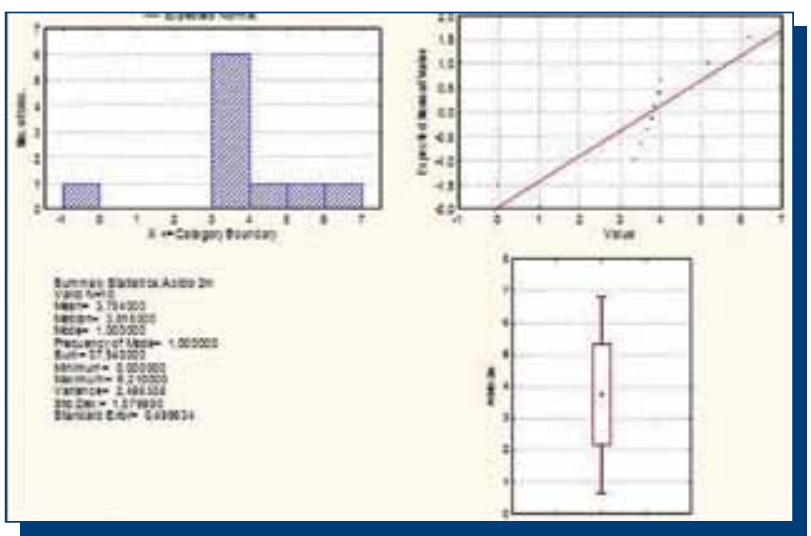

Gráfico 3. Histograma, distribución y Diagrama de Cajas (Boxplot) Grupo III.

Se evidencia una dispersión baja dentro de cada grupo, así como una simetría en la distribución de los Grupos I y III. El Grupo II posee una concentración de datos en un intervalo más amplio, pero con valores de resistencia más altos. El Grupo III muestra un intervalo muy bajo, pero con valores de resistencia más bajos.

\section{Análisis de Varianza (ANOVA) de un Factor}

Al aplicarse las pruebas de normalidad, el valor de $p$ indica una diferencia estadísticamente significativa entre el Grupo I (control) y Grupo II (ácido fluorhídrico por 1 minuto), no así entre el Grupo I (control) y el Grupo III (ácido fluorhídrico por 2 minutos), como muestra en las Tablas 3 y 4 .

Tabla 3. Resumen ANOVA

\begin{tabular}{|c|c|c|}
\hline \multicolumn{3}{|c|}{ Dependent: Acido $1 \mathrm{~m}$} \\
\hline Múltiple R: .69246700 & $\mathrm{~F}=7.370148$ \\
\hline $\mathrm{R}^{2}: .47951054$ & $\mathrm{df}=1.8$ \\
\hline No. of cases: 10 & adjusted R2: .41444936 & $\mathrm{p}=.026461$ \\
\hline \multicolumn{3}{|c|}{ Standard error of estimate: .846828206} \\
\hline Intercept: 6.556966127 & Std.Error: .7527798 & $\mathrm{t}(8)=8.7103$ \\
\hline
\end{tabular}

Tabla 4. Resumen ANOVA

\begin{tabular}{|c|c|c|}
\hline \multicolumn{3}{|c|}{ Dependent: Acido $2 \mathrm{~m}$} \\
\hline Múltiple $\mathrm{R}=.56671276$ & $\mathrm{~F}=3.784868$ \\
\hline $\mathrm{R}^{2}=.32116336$ & $\mathrm{df}=1.8$ \\
\hline No. of cases: 10 & adjusted $\mathrm{R}^{2}=.23630878$ & $\mathrm{p}=.087603$ \\
\hline \multicolumn{3}{|c|}{ Standard error of estimate: 1.380735765} \\
\hline Intercept: 1.522339291 & Std. Error: 1.227392 & $\mathrm{t}(8)=1.2403$ \\
\hline
\end{tabular}

\section{DISCUSIÓN}

El proceso de cementación reúne una serie de variables que actúan en conjunto, donde una falla en cualquiera de sus etapas lleva al fracaso de todo el procedimiento. El tipo de cementación (convencional o adhesiva) está directamente relacionada a la resistencia mecánica de la cerámica utilizada. Asimismo, debido a la poca o relativamente baja resistencia mecánica de las cerámicas ricas en matriz vítrea (porcelanas y vitro-cerámicas) se torna obligatorio el procedimiento de cementación adhesiva. Por lo tanto, para las cerámicas con alto contenido de cristales que presentan resistencia mecánica superior a las anteriores, una cementación convencional con cemento de fosfato de zinc, ionómero de vidrio y cemento resinoso puede ser ejecutada, aunque la cementación adhesiva sea siempre la opción más indicada.
El silano es una sustancia monomérica hidrolizable que compite con el agua en la superficie del vidrio formando una unión covalente entre el silicio del agente de unión y el oxígeno del grupo hidroxilo de la cerámica, proporcionando de este modo una adhesión química de la cerámica al cemento con base de resina y de éste a la estructura dental(49). No obstante, siendo una unión covalente, los resultados obtenidos en las muestras solamente silanizadas (3.35 Mpa) fueron los más bajos comparados con los demás tratamientos.

En las cerámicas con alto contenido de cristales, por la ínfima o completa ausencia de matriz vítrea, el proceso de cementación adhesiva se torna más complejo. Es en la matriz vítrea que encontramos la sílica, componente fundamental para la unión del silano. Siendo de esta manera que la silanización en estas piezas se torna ineficaz. Se debe enfatizar que la resistencia de unión entre una superficie condicionada sin la silanización es menor en torno de $65 \%$ que en las superficies silanizadas $^{(50,51)}$. En adición, el acondicionamiento con ácido fluorhídrico estándar para las categorías anteriores de cerámicas, también se torna despreciable, ya que este ácido no tiene poder para condicionar los cristales cerámicos, solamente condicionan la matriz vítrea. Por lo tanto, en este tipo de cerámicas, debemos elegir un método de tratamiento de superficie diferente al ácido fluorhídrico.

El protocolo estándar cementar restauraciones cerámicas feldespáticas requiere grabado ácido y tratamiento con silano sobre la superficie de la porcelana ${ }^{(52)}$. El grabado ácido de la porcelana se ha utilizado ampliamente para mejorar la retención entre el cemento de resina y la restauración. Algunos estudios han sugerido que el grabado se realiza preferentemente con ácido fluorhídrico en vez de ácido fosfórico ${ }^{(52,53)}$. No obstante, el ácido fosfórico puede mejorar la energía superficial, por la limpieza de la superficie cerámica ${ }^{(54)}$. El grabado de cerámica es un proceso dinámico y el impacto depende de la constitución del sustrato, la topografía de la superficie, la concentración del ácido y tiempo de grabado ${ }^{(30,55)}$. En cuanto al tiempo de grabado, se han realizado múltiples estudios con diferentes tipos de cerámica y concentraciones de ácido fluorhídrico ${ }^{(26,27,55,56)}$. Chen y al. ${ }^{(26)}$ evaluaron dos concentraciones de ácido $(2.5$ y $5 \%$ ) y siete tiempos diferentes de grabado $(0,30,60,90$, 120,150 y 180 segundos). Períodos de grabado por encima de los 30 segundos mejoran de manera efectiva la fuerza de adhesión al cemento de resina. Los autores concluyeron que el grabado con ácido fluorhídrico por 120 s presenta una fuerza de unión adecuada de la porcelana al cemento de resina.

Se sabe que el grabado con ácido fluorhídrico proporciona la rugosidad de la superficie cerámica necesaria para mejorar las propiedades mecánicas, pero el grabado por tiempos excesivos podría tener un efecto adverso, debilitando la porcelana ${ }^{(55,57,58)}$. Por lo tanto, es importante conocer el tiempo adecuado de grabado para lograr una adecuada retención micromecánica sin debilitar la cerámica. Desde que el grabado con ácido fluorhídrico se sugirió por primera vez como tratamiento de la superficie cerámica para la unión con el cemento de resina, muchas combinaciones diferentes entre tiempos de grabado y la concentración de ácido se han propuesto y utilizado ${ }^{(25-27,30,53,54,56,59-62)}$. Wolf y al. ${ }^{(62)}$, evaluó la rugosidad de superficie de una porcelana feldespática grabada con ácido fluorhídrico al 9.5\% para 30, 60, 150 y 300 segundos, y la resistencia de la unión a la tracción a un cemento de resina. Los autores encontraron una correlación positiva entre rugosidad de la superficie de cerámica y aumentar el tiempo de grabado con ácido fluorhídrico, el cual coincide con otros estudios ${ }^{(30,60)}$

Se ha sugerido por los autores de que la concentración de ácido y el tiempo de grabado tienen un efecto profundo en la modificación de la superficie de la porcelana feldespática. El debilitamiento es debido a la extensión de los defectos existentes en la superficie de la porcelana por la variación en el tiempo de grabado y la concentración del ácido, alterando la naturaleza y distribución de los defectos, de modo que el debilitamiento de la unión al cemento de resina ${ }^{(63)}$ se traduce en una disminución del rendimiento clínico. Por lo tanto, los autores proponen que la longevidad de las restauraciones de porcelana puede ser influenciada por la variación en la concentración y el tiempo de grabado con ácido fluorhídrico. Sin embargo, no se puede suponer que diferentes marcas o tipos de porcelana mostrarán exactamente el mismo patrón de resistencia y fiabilidad después de la aplicación de ácido fluorhídrico en diferentes concentraciones y tiempos de grabado. Diferentes materiales pueden ser más o menos sensibles al ácido fluorhídrico, lo que sugiere la necesidad de otros estudios similares a los reportados aquí.

De acuerdo con la mayoría de los estudios consultados y en concordancia con este experimento, el mejor tratamiento de superficie para las cerámicas ricas en matriz vítrea (porcelanas y vitro-cerámicas) 
sería el uso de ácido fluorhídrico al $10 \%$, asociado a la silanización. En estos trabajos los tiempos de aplicación del ácido fluorhídrico fluctuaron entre 1 y 3 minutos ${ }^{(64,65)}$, dependiendo de la cerámica utilizada, no debiendo ser superior a este intervalo, pues tiempos adicionales resultarían en una disminución de la resistencia de unión ${ }^{(65)}$.

De esta forma, las muestras tratadas con ácido fluorhídrico por 1 minuto (Grupo II) presentaron una unión (4.64 MPa) estadísticamente mayor que el Grupo control sólo silanizada, pero las muestras tratadas con ácido fluorhídrico por 2 minutos (Grupo III) presentaron resultados que no difirieren estadísticamente del Grupo control.

\section{CONCLUSIONES}

Dentro de las limitaciones del presente estudio in vitro, y a la vista de los resultados proporcionados, tanto por el análisis estadístico realizado como por la observación, podemos llegar a las siguientes conclusiones:

- El método de tratamiento superficial con ácido fluorhídrico promueve microretenciones, con lo cual aumentan significativamente los valores de resistencia de unión a la tracción, lo que puede traducirse en un aumento de beneficio clínico. Esto se evidencia en las muestras tratadas con ácido fluorhídrico por 1 minuto, las cuales presentan valores de resistencia estadísticamente superiores a la muestra de control.
- Un tiempo excesivo de acondicionamiento con ácido fluorhídrico promueve la completa disolución de la matriz vítrea alrededor de los cristales, provocando el dislocamiento de los mismos, disminuyendo las retenciones micromecánicas y fragilizando la restauración protética.

- Para este tipo de porcelana, y según los resultados obtenidos, el tiempo de grabado con ácido fluorhídrico por 2 minutos puede resultar excesivo, ya que si bien mejora los valores comparados a las muestras sin acondicionar, no presenta diferencias estadísticamente significativas con estas. Por lo tanto, se rechaza la hipótesis planteada.

- Los resultados aquí encontrados, no pueden ser extrapolados para otros sistemas cerámicos, los cuales poseen composición y porcentaje de fase vítrea y cristalina diferentes del material aquí estudiado. Investigaciones adicionales son necesarias para definir los protocolos recomendados para la cementación adhesiva de estos materiales cerámicos considerando otras variables e instrumentos de estudio, como por ejemplo incluir un análisis bajo microscopía electrónica, o midiendo ángulo de contacto sobre las superficies tratadas. También se sugiere complementar este estudio comparando diferentes tipos de tratamiento de superficie y con diferentes tiempos de grabado sobre diferentes tipos de cerámica.

\section{CONFLICTOS DE INTERÉS}

Los autores declaran no tener conflictos de interés.

\section{REFERENCIAS BIBLIOGRÁFICAS}

1. Saravia M. Adhesión a sustratos no dentarios. En: Gilberto Henostroza. Operatoria Dental y Biomateriales 2000. $1^{\text {ra }}$ ed. Lima Perú: Multi-impresos; 2000.

2. Saavedra M. Procedimientos clínicos para carillas. En: Gilberto Henostroza. Operatoria Dental y Biomateriales 2000. $1^{\text {ra }}$ ed. Lima Perú: Multi-impresos; 2000.

3. Cho GC, Donovan TE, Chee WW. Clinical experiences with bonded porcelain laminate veneers. J Calif Dent Assoc, 1998; 26: 121-127.

4. Dumfahrt $H$, Schaffer $H$. Porcelain laminate veneers. A retrospective evaluation after 1 to 10 years of service: Part II-clinical results. Int J Prosthodont, 2000; 13: 9-18. 5. Friedman MJ. Ask the experts: Porcelain veneers. J Esthet Restor Dent, 2001; 13: $86-87$.

6. Fradeani M. Six-year follow-up with empress veneers. Int $J$ Periodontics Restorative Dent, 1998; 18: 216-225

7. Peumans M, Van Meerbeek B, Lambrechts P, Vanherle G. Porcelain veneers: A review of the literature. J Dent, 2000; 28: 163-177.

8. Blatz MB. Long-term clinical success of all-ceramic posterior restorations. Quintessence Int, 2002; 33: 415-426.

9. Felden A, Schmalz G, Federlin M, Hiller KA. Retrospective clinical investigation and survival analysis on ceramic inlays and partial ceramic crowns: Results up to 7 years. Clin Oral Investig, 1998; 2: 161-167.

10. van Dijken JW, Hoglund-Aberg C, Olofsson AL. Fired ceramic inlays: A 6-year follow up. J Dent, 1998; 26: 219-225.

11. Hayashi M, Tsuchitani Y, Miura M, Takeshige F, Ebisu S. 6-year clinical evaluation of fired ceramic inlays. Oper Dent, 1998; 23: 318-326.

12. Fuzzi M, Rappelli G. Ceramic inlays: Clinical assessment and survival rate. $J$ Adhesive Dent, 1999; 1: 71-79.

13. Roulet JF. Longevity of glass ceramic inlays and amalgam-results up to 6 years. Clin Oral Invest, 1997; 1: 40-46.

14. Mormann W, Krejci I. Computer-designed inlays after 5 years in situ: Clinical performance and scanning electron microscopic evaluation. Quintessence Int, 1992; 23: 109-115.

15. Pallesen $U$. Clinical evaluation of CAD/CAM ceramic restorations: 6-year report. In: Mormann WH, editor. CAD/CIM in aesthetic dentistry: CEREC 10 year anniversary symposium. Berlin: Quintessence; 1996. p. 241-253.

16. Berg NG, Derand T. A 5-year evaluation of ceramic inlays (CEREC). Swed Dent J, 1997; 21: 121-127.

17. Sjogren G, Molin M, van Dijken JW. A 5-year clinical evaluation of ceramic inlays (Cerec) cemented with a dual-cured or chemically cured resin composite luting agent. Acta Odontol Scand, 1998; 56: 263-267.

18. el-Mowafy $\mathrm{O}$, Rubo MH. Resin-bonded fixed partial dentures-a literature review with presentation of a novel approach. Int J Prosthodont, 2000; 13: 460-467.

19. Corrente G, Vergnano L, Re S, Cardaropoli D, Abundo R. Resin-bonded fixed partial dentures and splints in periodontally compromised patients: A 10-year followup. Int J Periodontics Restorative Dent, 2000; 20: 628-636.

20. Behr M, Leibrock A, Stich W, Rammelsberg P, Rosentritt M, Handel G. Adhesive-fixed partial dentures in anterior and posterior areas: Results ofan ongoing prospective study begun in 1985. Clin Oral Investig, 1998; 2: 31-35.

21. Kern M, Strub JR. Bonding to alumina ceramic in restorative dentistry: Clinical results over up to 5 years. J Dent, 1998; 26: 245-249.

22. Malament KA, Socransky SS. Survival of Dicor glass-ceramic dental restorations over 14 years: Part I: Survival of Dicor complete coverage restorations and effect of internal surface acid etching, tooth position, gender, and age. J Prosthet Dent, 1999; 81: 23-32.

23. Malament KA, Socransky SS. Survival of Dicor glass-ceramic dental restorations over 14 years. Part II: Effect of thickness of Dicor material and design of tooth preparation. J Prosthet Dent, 1999; 81: 662-667.

24. Malament KA, Socransky SS. Survival of Dicor glass-ceramic dental restorations over 16 years. Part III: Effect of luting agent and tooth or tooth-substitute core structure. J Prosthet Dent, 2001; 86: 511-519.

25. Hooshmand T, Parvizi S, Keshvad A. Effect of surface acid etching on the biaxial flexural strength of two hot-pressed glass ceramics. J Prosthodont, 2008; 17: 415-419. 26. Chen $\mathrm{JH}$, Matsumura $\mathrm{H}$, Atsuta $\mathrm{M}$. Effect of etchant, etching period, and silane priming on bond strength to porcelain of composite resin. Oper Dent, 1998; 23: 250-257. 27. Chen $\mathrm{JH}$, Matsumura $\mathrm{H}$, Atsuta M. Effect of different etching periods on the bond strength of a composite resin to a machinable porcelain. J Dent, 1998; 26: 53-58.

28. Jardel V, Degrange M, Picard B, Derrien G. Surface energy of etched ceramic Int J Prosthodont, 1999; 12: 415-418.

29. Yen TW, Blackman RB, Baez RJ. Effect of acid etching on the flexural strength of a feldspathic porcelain and a castable glass ceramic. J Prosthet Dent, 1993; 70 : 224-233.

30. Addison O, Marquis PM, Fleming GJ. The impact of hydrofluoric acid surface treatments on the performance of a porcelain laminate restorative material. Dent Mater, 2007; 23: 461-468.

31. Chang JC, Hart DA, Estey AW, Chan JT. Tensile bond strenghts of five luting agents to two CAD-CAM restorative materials and enamel. $J$ Prosthet Dent, 2003; 90: $18-23$.

32. el-Mowafy $\mathrm{O}$. The use of resin cements in restorative dentistry to overcome retention problems. J Can Dent Assoc, 2001; 67: 97-102.

33. Sorensen JA, Kang SK, Avera SP. Porcelain-composite interface microleakagewith various porcelain surface treatments. Dent Mat, 1991; 7: 118-123 34. Jensen ME, Sheth JJ, Tolliver D. Etched-porcelain resin-bonded fullveneer crowns: In vitro fracture resistance. Compendium, 1989; 10: 336-338, 340-341, 344 347

35. McLean JW, Hughes TH. The reinforcement of dental porcelain with ceramic oxides. Br Dent J, 1965; 119: 251-267.

36. Seghi RR, Sorensen JA. Relative flexural strength of six new ceramic materials Int J Prosthodont, 1995; 8: 239-246.

37. Andersson M, Oden A. A new all-ceramic crown: A dense-sintered, high-purity alumina coping with porcelain. Acta Odontol Scand, 1993; 51: 59-64.

38. Zeng K, Oden A, Rowcliffe D. Flexure tests on dental ceramics. Int J Prosthodont 1996; 9: 434-439.

39. Zeng K, Oden A, Rowcliffe D. Evaluation of mechanical properties of dental ceramic core materials in combination with porcelains. Int J Prosthodont, 1998; 11: 183-189. 40. Ashizuka M, Kiyohara H, Okuno T, Kubota Y. Fatigue behavior oftetragonal zirconia polycrystals (Y-TZP) containing 2 and 4 mol\% Y2O3 (Part 2). J Ceram Soc Jpn Inter Ed, 1988; 96: 731-736.

41. Taira M, Nomura Y, Wakasa K, Yamaki M, Matsui A. Studies on fracture toughness of dental ceramics. J Oral Rehabil, 1990; 17: 551-563.

42. Giordano RA 2nd, Pelletier L, Campbell S, Pober R. Flexural strength of an infused ceramic, glass ceramic, and feldspathic porcelain. J Prosthet Dent, 1995; 73: $411-418$

43. Stangel I, Nathanson D, Hsu CS. Shear strength of the composite bond to etched porcelain. J Dent Res, 1987; 66: 1460-1465.

44. Paffenbarger GC, Sweeney WT, Bowen RL. Bonding porcelain teeth to acrylic resin denture bases. J Am Dent Assoc, 1967; 74: 1018-1023.

45. Newburg R, Pameijer $\mathrm{CH}$. Composite resins bonded to porcelain with silane solution. J Am Dent Assoc, 1978; 96: 288-291. 
46. Kern M, Neikes MJ, Strub JR. Tensile strength of the bond to In-Ceram after varying modes of surface conditioning. Dtsch Zahnarztl Z, 1991; 46: 758-761.

47. Kern M, Thompson VP. Sandblasting and silica coating of a glassinfiltrated alumina ceramic: Volume loss, morphology and changes in surface composition. $J$ Prosthet Dent, 1994; 71: 453-461.

48. Roulet JF, Soderholm KJM, Longmate J. Efect of treatment and storage conditions on ceramic/composite bond strenght. J Dent Res, 1995; 74: 381-387. 49. van Noort R. Introduction to Dental Materials. London, Mosby. 1994.

50. Meiners H, Herrmann R, Spitzbarth S. ZurVerbundfestigkeitdesRocatecSystems. Dental Labor, 1990; 38(2): 185-188.

51. May KB, Fox J, Razzoog ME, Lang BR. Silane to enhance the bond between polymethyl methacrylate and titanium. J Prosthet Dent, 1995; 73(5): 428-431.

52. Stewart GP, Jain P, Hodges J. Shear bond strength of resin cements to both ceramic and dentin. J Prosthet Dent, 2002; 88: 277-284.

53. Klosa K, Wolfart S, Lehmann F, Wenz HJ, Kern M. The effect of storage conditions, contamination modes and cleaning procedures on the resin bond strength to lithium disilicate ceramic. J Adhes Dent, 2009; 11: 127-135.

54. Canay S, Hersek N, Ertan A. Effect of different acid treatments on a porcelain surface. J Oral Rehabil, 2001; 28: 95-101.

55. Della Bona A, Anusavice KJ, Hood JA. Effect of ceramic surface treatment on tensile bond strength to a resin cement. Int J Prosthodont, 2002; 15: 248-255.

56. Guler AU, Yilmaz F, Yenisey M, Guler E, Ural C. Effect of acidetching time and a self-etching adhesive on the shear bond strength of composite resin to porcelain J Adhes Dent, 2006; 8: 21-25.
57. Della Bona A, Van Noort R. Shear vs. tensile bond strength of resin composite bonded to ceramic. J Dent Res, 1995; 74: 1591-1596.

58. Addison O, Fleming GJ. The influence of cement lute, thermocycling and surface preparation on the strength of aporcelain laminate veneering material. Dent Mater, 2004; 20: 286-292.

59. Della Bona A, Anusavice KJ. Microstructure, composition, and etching topography of dental ceramics. Int J Prosthodont, 2002; 15: 159-167.

60. Chaiyabutr Y, McGowan S, Phillips KM, Kois JC, Giordano RA. The effect of hydrofluoric acid surface treatment and bond strength of a zirconia veneering ceramic. J Prosthet Dent, 2008; 100: 194-202.

61. Naves LZ, Soares CJ, Moraes RR, Gonçalves LS, Sinhoreti MA, CorrerSobrinho L. Surface/interface morphology and bond strength to glass ceramic etched for different periods. Oper Dent, 2010; 35: 420-427.

62 . Wolf DM, Powers JM, O'Keefe KL. Bond strength of composite to etched and sandblasted porcelain. Am J Dent, 1993; 6: 155-158.

63. Della Bona A, van Noort R. Ceramic surface preparations for resin bonding. Am J Dent, 1998; 11: 276-280

64. Stangel I, Nathanson D, Hsu CS. Shear strength of the composite bond to etched porcelain. J Dent Res, 1987; 66(9): 1460-1465

65. Wolf DM, Powers JM, O'Keefe KL. Bond strength of composite to etched and sandblasted porcelain. Am J Dent, 1993; 6(3): 155-158. 